\title{
ECN-capable TCP-friendly Layered Multicast Multimedia Delivery
}

\author{
Robert R. Chodorek \\ Department of Telecommunications \\ The AGH University of Science \\ and Technology \\ Kraków, Poland \\ chodorek@kt.agh.edu.pl
}

\author{
Agnieszka Chodorek \\ Department of Telecommunications \\ and Photonics \\ Kielce University of Technology \\ Kielce, Poland \\ a.chodorek@tu.kielce.pl
}

\begin{abstract}
ECN-capable TCP-friendly layered multicast multimedia delivery combines explicit congestion notification (ECN) with the TCP-friendly layered multicast scheme. TCP-Friendliness denotes that receiverdriven architecture behaves under congestion like the TCP protocol. Unlike well-known TCP-friendly protocols (TFRC, TFMCC, WEBRC), which use TCP throughput the equation, our system assures TCPfriendliness by emulation. The Receiver emulates TCP congestion control mechanism working with ECN congestion notification. Decisions about changing group membership and, as a result, about changing effective transmission rate, are taken based on TCP emulation. In the paper, a simulation model of ECN-capable multicast multimedia delivery in ns-2 environment is discussed.
\end{abstract}

\section{Introduction}

TCP-friendliness is the ability of a system to behave under congestion like the Transmission Control Protocol (TCP) [1]. TCP-friendly systems vary sending rate in response to congestion in the same way TCP does. Usually, TCP-friendly systems send data at a rate calculated from the TCP throughput equation.

TCP-friendliness is the recommended behavior for new transport protocols. The well-known TCP-friendly protocols are: TCP Friendly Rate Control (TFRC) [2], TCP-Friendly Multicast Congestion Control (TFMCC) [3], Wave and Equation Based Rate Control (WEBRC) [4] and Adaptive Smooth Multicast Protocol (ASMP) [5][6]. TCP-friendly behavior can also manifest other congestion control architectures, including receiverdriven layered multicast [7][8][9].

The aim of the paper is to propose TCP-friendly congestion control for multimedia delivery, based on ECN-capable RTP and layered multicast architecture.
The proposed solution doesn't require additional protocol (for example RLM) to manage transmission. In contrast to typical TCP-friendly solutions, which use the TCP-throughput equation or the leaky bucket algorithm, our solution directly emulates the ECN-capable TCP congestion control algorithm.

The paper is organized as follows. Section 2 describes TCP congestion control. Section 3 proposes ECN-capable TCP-friendly layered multicast. Section 4 presents an analysis of the proposed solution. Section 5 summarizes our experiences.

\section{The congestion control mechanism used in the TCP protocol}

The congestion control mechanism, used in the TCP transport protocol, is a superposition of four algorithms [1]: slow start, congestion avoidance, fast retransmit and fast recovery. The congestion control mechanism is based on a window - the sender cannot send more data than is allowed by the size of the window.

Window-based control was introduced to the TCP in the RFC 793. The RFC defines a receiver window (rwnd), which is used to the flow control. The size of the receiver window is set by the TCP receiver and is sent to the sender - first time, during the connection establishment, and next in every ACK packet. Typically, the size of the receiver window changes slowly.

The congestion control mechanism introduces to the TCP a second window - the so-called congestion window (cwnd). In contrast to the receiver window, the congestion window evolves continuously. It is decremented during congestion and incremented if congestion is not detected. Rate of incrementing depends on probability of congestion. If the probability of congestion is small, size of the congestion window will grow exponentially, according to the slow start algorithm. If the probability of congestion is large, the size of the congestion window will grow linearly, according to the 
congestion avoidance algorithm. The boundary between a small and large probability of congestion is defined by the so-called slow start threshold (ssthresh). Typically, the initial value of ssthresh is equal to rwnd.

During the slow start phase, the congestion window is incremented by 1 after receiving the ACK packet:

$$
\text { cwnd }_{\mathrm{k}+1}=\mathrm{cwnd}_{\mathrm{k}}+1 \text {, }
$$

During the congestion avoidance phase, the congestion window is incremented by 1 after receiving a whole non-overlapping window. In practice:

$$
\mathrm{cwnd}_{\mathrm{k}+1}=\operatorname{cwnd}_{\mathrm{k}}+\frac{1}{\mathrm{cwnd}_{\mathrm{k}}} \text {. }
$$

The TCP window $(w)$, which limits the amount of non-acknowledged data in the transmission system, is the smallest value from rwnd and cwnd:

$$
\mathrm{w}=\min (\mathrm{cwnd}, \mathrm{rwnd}) \text {. }
$$

If congestion appears, the congestion window is decremented. In the early stages, congestion is detected by TCP receivers as a gap in sequence space or $\mathrm{ECN}$ signaling. The new value of cwnd is then set to:

$$
\mathrm{Cwnd}=\frac{1}{2} \mathrm{~W}
$$

If congestion increases it is detected by the sender based on the retransmission timer. The new value of cwnd is then set to:

$$
\text { Cwnd }=1
$$

After each decrementing of cwnd, the ssthresh is set to a half of current $w$ size:

$$
\text { sstresh }=\max \left(2, \frac{1}{2} \mathrm{w}\right),
$$

\section{TCP-friendly layered multicast}

In the paper, we propose ECN-capable TCPfriendly layered multicast. The proposed solution combines receiver-driven layered multicast architecture with ECN-capable RTP protocol and TCP-friendly congestion control.

\subsection{Receiver-driven layered multicast}

Layered multicast is a transmission where a highquality multimedia stream is encoded into complementary sub-streams, so-called layers. Layers are strictly synchronized and simultaneously transmitted through the network as separate multicast groups. Usually, layers are encoded in a cumulative manner and, at a given time, one receiver subscribes to the base layer (guaranteed the lowest level of quality with the minimal bandwidth requirements) and zero or more supplemen- tary layers (to improve quality). Layers can be joining/leaving only in order of their relevance.

If congestion appears in the network, the receiver will leave the highest received layer that leads to reduction of consumption of network resources. Otherwise, the receiver can subscribe to the next layer and improve the quality of delivered multimedia. The decision about the number of received layers is taken autonomously by each receiver.

\subsection{ECN-capable RTP protocol}

ECN-capable RTP protocol was defined in [10]. It is an extension of the RTP protocol, able to convey Explicit Congestion Notification (ECN) signaling.

ECN-capable RTP sender sets the ECN bits in the IP header to indicate the ECN-capability of the transport protocol end-point. The Packets with ECNCapable Transport codepoint $\mathrm{CE}(0)$ are sent to the receiver(s) using unicast or multicast transmission. If the congestion builds up, routers on the delivery tree will set the Congestion Experienced (CE) in the IP header. As a result, the RTP receiver gets the information about congestion appearing in at least one network node along the delivery tree.

If the receiver obtains a congestion indication (a single CE packet), the feedback to the sender will be sent using a new type of RTCP message - ECNfeedback (EFB). RTP with ECN-capable congestion control can work both in sender-driven or receiverdriven architecture. The sender-driven one can utilize the EFB message. The receiver-driven one can hold the EFB messages and perform congestion control itself.

\subsection{TCP-friendly layered multicast}

TCP-friendly layered multicast combines receiverdriven layered multicast transmission with TCPfriendly congestion control. In the proposed solution, we directly implement the ECN-capable TCP congestion control algorithm.

3.3.1. Increasing transmission rate. If congestion isn't detected, the TCP increases the transmission rate. Because layered multicast transmission requires a continuous join to the base layer, we decided to omit the slow start phase. Simulation results, presented in Section 4, show, that this decision hasn't had a significant influence on the TCP-friendly behavior of our system. The TCP reacts to lack of congestion by a linear increasing of the congestion window. The window is incremented by 1 after receiving a whole non-overlapping window - i.e. after the Round Trip Time (RTT). Because real-time transmission cannot be window con- 
trolled in our system, we emulate this mechanism by increasing a BW variable. This variable represents TCP throughput, which will be achieved if the current TCP congestion window will be incremented by 1 :

$$
\mathrm{BW}_{\mathrm{k}+1}=\left(\mathrm{cwnd}_{\mathrm{k}}+1\right) \cdot \frac{M S S}{R T T} \text {. }
$$

Thus:

$$
\mathrm{BW}_{\mathrm{k}+1}=\mathrm{BW}_{\mathrm{k}}+\frac{M S S}{R T T} .
$$

If BW exceeds the sum of the bit rate of the currently joined layers and the bit rate of the next layer, the receiver will join the next layer.

3.3.2. Decreasing transmission rate. If the transmission system detects congestion, the TCP decreases the transmission rate. Because layered multicast transmission requires a continuous join to the base layer, our system cannot decrease transmission rate below the bit rate of the base layer.

The TCP reacts to congestion by decreasing the congestion window according to equation (4). The general ECN-capable receiver behaviour, recommended in RFC 3168, suggests that the response to the obtained CE codepoint must be essentially the same as the response to a single dropped packet. Thus, the ECN signaling doesn't significantly affect the behavior of the proposed system, but allows the system to avoid unnecessary packet loses. It is especially important in the case of the RTP transmission, which isn't error controlled, but error rates should be minimal.

In the proposed system, we emulate decreasing the $\mathrm{TCP}$ congestion window by decreasing a $\mathrm{BW}$ variable:

$$
B W_{k+1}=\frac{B W_{k}}{2} \text {. }
$$

If BW is less than the sum of the bit rates of currently joined layers, the receiver will leave as many layers as allowed by the BW variable.

3.3.3. RTT estimation. To properly estimate the TCP throughput, an accurate estimation of the RTT time is necessary. Unfortunately, during multicast transmission, accurate estimation of the RTT is possible only in a homogeneous environment. In the case of a heterogeneous environment, where each path between the sender and one of the receivers can have different delay, proper estimation of RTT is very difficult and sometimes impossible.

One possible solution is to use a designated receiver (sometimes called acker), which estimates the RTT. This solution is used, for example, in TFRC and TFMCC protocols. However, because the worst-case receiver is usually set to be the designated receiver, this method allows for the estimation of RTT only in the worst-case path (the most congested path).

In our solution, we estimate RTT by using a modification of the Multicast Round Trip Time (MRTT) applied in the WEBRC protocol [4]. The MRTT is measured as the time between when a join IGMP (or MLD) packet is sent and when the first multicast packet from the joined group is received. MRTT is measured by every receiver for each join operation. As a result, each receiver has it's own MRTT estimation.

\section{Simulations}

All simulations described in the paper were carried out using an event-driven ns-2 simulator [11], developed in U. C. Berkeley. Elastic traffic was modeled using a build-in model of ftp transmission. Inelastic traffic originates from external files, which contain Constant Bit Rate (CBR) traces.

\subsection{Experiments}

Experiments have been carried out using typical single-bottleneck topologies, which consist of two or three routers and a set of senders and receivers. In all experiments throughput of the bottleneck link was large enough to assure live video transmission.

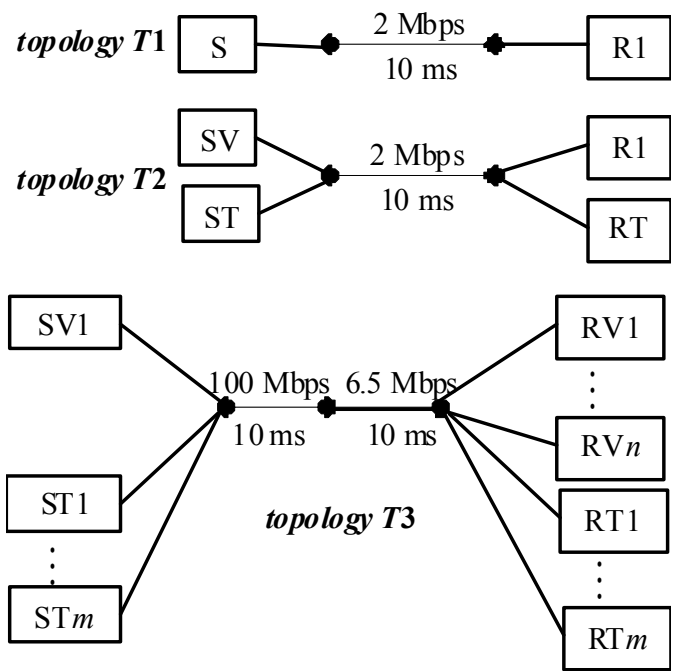

Figure 1. Topologies used in experiments.

The transmission scheme has been simulated in 3 different topologies (Fig. 1), to explore the performance issues of the proposed solution.

Topology T1 consists of a single source (S) of elastic (FTP) or inelastic (CBR) traffic and single receiver (R1). Topology T2 consists of a video source (SV), a TCP source (ST), video receiver (RV), and TCP receiver (RT). Topology T3 extends topology T2 with 
multiple video receivers and multiple FTP sessions. The number of FTP senders/receivers was set to 10 and number of video receivers was also set to 10 .

Senders were connected to the nearest router at 100 Mbps and 1ms delay. In the router, a Drop Tail queue was used and the queue size was set to 5000 packets that, in practice, give an infinite buffer size (topologies $T 1$ and $T 3$ ). In the case of topology $T 2$, the RED queue was used. Senders' buffers are theoretically unlimited.

Receivers were connected to the nearest router at 10 Mbps and 5 ms delay. In the router, RED queue was used to avoid synchronization (topologies $T 1, T 2, T 3$ ).

Core routers were connected with a bottleneck link at $2 \mathrm{Mbps}$ (topology $T 1$ and T2) and 6.5 Mbps (topology T3) and propagation delay of $10 \mathrm{~ms}$. The intermediate router (topology T3) used the RED queue. In all experiments, RED queues use ECN signaling (their mark packets instead of drops).

As the source of elastic traffic (ST), FTP over TCP (SACK version) was used. TCP packets had a maximum segment size (MSS) of 1000 bytes. As a model of video (inelastic traffic), constant bit rate (CBR) streams were used. The CBR stream was transmitted using ECN-capable RTP [10]. The maximum payload size of RTP packets was set to 200 bytes (small packets) or 1000 bytes (large packets).

\subsection{Responsiveness to packet loss rate}

In this experiment we investigate how TCP-friendly layered multicast responds to changes in the loss rate and evaluate its performance. In our simulations we use topology T1. Because we want to answer the question about boundaries of usage of our solution to multimedia transmission, all experiments were carried out in dedicated link (no background traffic was used).

Fig. 2 depicts throughput of selected transmissions as a function of packet error rate. In the experiment we compare ECN-capable TCP-friendly layered multicast with TCP and TFRC transmissions. In all experiments we use packet size of 1000 B (Fig. 2a) and 200 B (Fig. $2 b$ and Fig. 2c).

ECN-capable TCP-friendly layered multicast transmissions were carried out for 3,6 and 10 layers. TCP transmissions were carried out for FTP over TCP (both packet drops and ECN signaling). TFRC transmissions were carried out for video over TFRC (1.2 Mbps CBR stream, ECN signaling).

Generally, ECN-capable TCP-friendly layered multicast isn't more aggressive than the TCP and, in some situations, than the TFRC. If we use large number of layers, the transmission will be more aggressive than if we use small number of layers. However, it never exceeds boundary of ECN-capable TCP transmission.
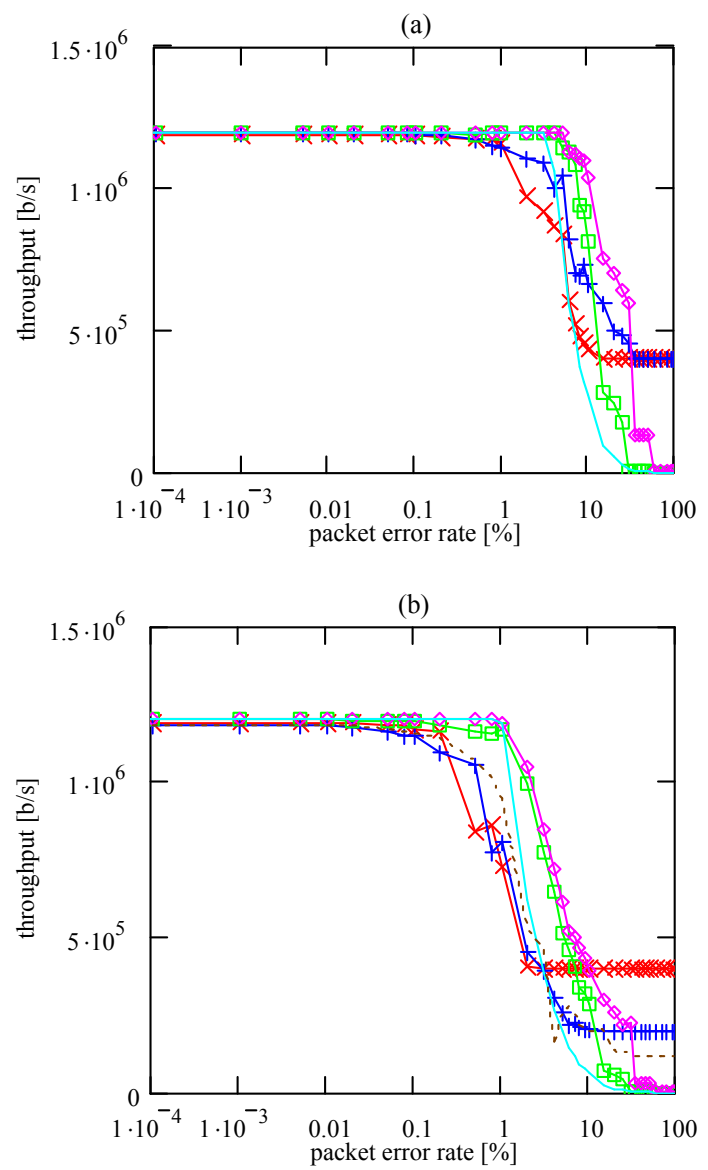

(c)

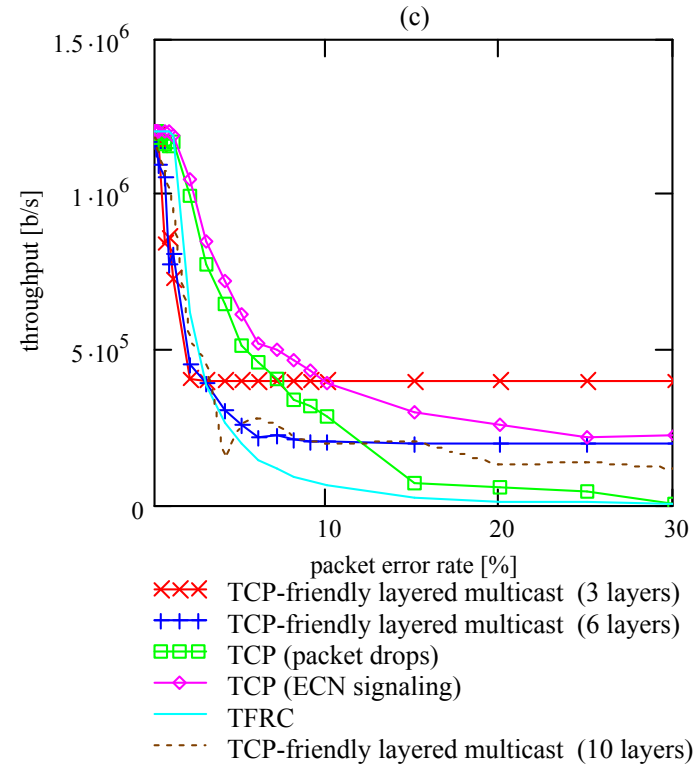

Figure 2. Throughput as a function of packet error rate: a) packet's size $1000 \mathrm{~B}, \mathrm{~b}, \mathrm{c})$ packet's size $200 \mathrm{~B}$, logarithmic (b) and linear (c) scale. 


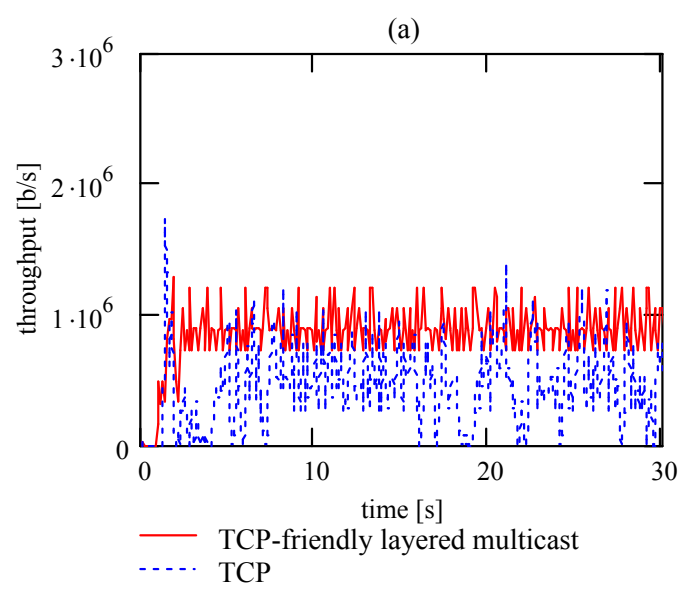

(b)

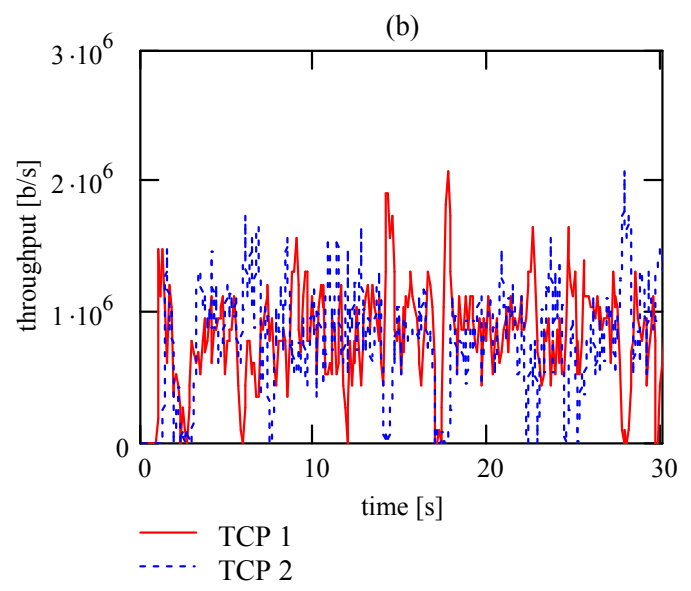

(c)

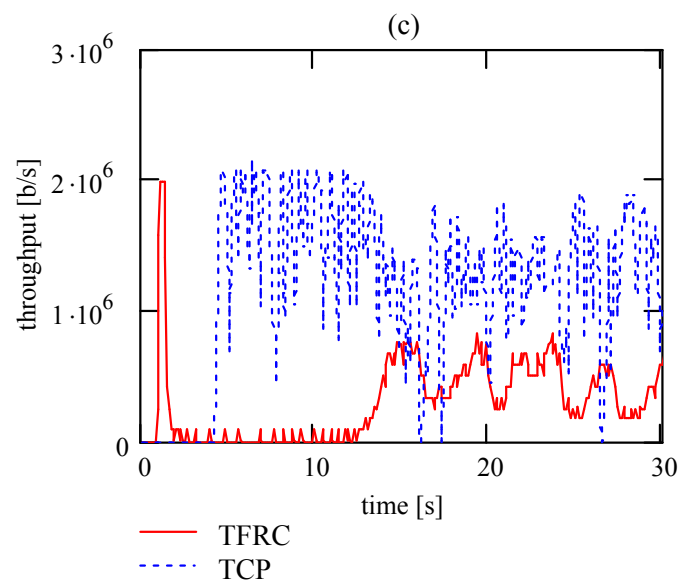

Figure 3. FTP over TCP (dotted line) competes for bandwidth with video stream (solid line): a) TCP vs. TCP-friendly layered multicast (3 layers), b) TCP vs. CBR over TCP, c) TCP vs. CBR over TFRC. Parameters of video source: CBR, 1.2 Mbps.
In the case of large packets (1000 B), a small number of layers causes that graph of throughput vs. packet error rate to be close to the graph for TFRC. A large number of layers causes that proposed solution to be more aggressive than the TFRC. In the case of small packets $(200 \mathrm{~B})$, there are no significant differences between small and large numbers of layers. However, a larger number of layers gives a more smoother transmission.

\subsection{Responsiveness to competing traffic}

In this experiment we investigate how ECN-capable TCP-friendly layered multicast responds to competing elastic traffic. In our simulations we use topology T2.

Fig. 3 depicts throughput of FTP over TCP (with elastic traffic), which competes for bandwidth (2 Mbps) with inelastic traffic. In the experiment we compare ECN-capable TCP-friendly layered multicast (3 layers) with video (CBR) over TCP and video over TFRC transmissions. Packet size was set to $1000 \mathrm{~B}$.

As is shown in Fig. 3, the proposed solution both preserve real-time character of the video stream and is able to fairly share bandwidth with competing TCP flow. As we expected, the video over TCP is able to fairly share bandwidth, but isn't able to realize video transmission in real-time. Surprisingly, exemplary video over TFRC transmission, depicted in Fig. 3c, isn't able to preserve real-time character of the video stream and isn't aggressive enough to achieve fair bandwidth allocation.

\subsection{Fairness}

In this experiment we investigate if ECN-capable TCP-friendly layered multicast is able to achieve fairness towards competing TCP traffic. In our simulations we use topology T3. Proposed ECN-capable TCPfriendly layered multicast competed for $6.5 \mathrm{Mbps}$ link with 10 FTP over TCP flows. As a result, each of 11 transmissions (10 TCP plus 1 aggregated RTP transmissions) should occupy $1 / 11$ of $6.5 \mathrm{Mbps}$, i.e. 0.59 Mbps.

Fig. 4 depicts throughput of exemplary FTP over TCP (with elastic traffic), which competes for bandwidth with inelastic traffic. In the experiment we use ECN-capable TCP-friendly layered multicast transmission (6 layers), which was carried out from one sender to 10 receivers. Both TCP and RTP packet sizes were set to $200 \mathrm{~B}$.

As is shown in Fig. 4, the proposed solution is able to be reasonably fair (with the factor of 2 [2]) in sharing bandwidth with the TCP. Detailed results are as follows: ECN-capable TCP-friendly layered multicast 
transmission occupies $0.843 \mathrm{Mbps}$. 10 FTP over TCP transmissions occupy $0.53 \mathrm{Mbps}, 0.51 \mathrm{Mbps}, 0.57$ Mbps, 0.48 Mbps, 0.49 Mbps, 0.55 Mbps, 0.49 Mbps, $0.6 \mathrm{Mbps}, 0.57 \mathrm{Mbps}$, and 0.5 Mbps. Mean throughput of FTP over TCP is equal to $0.53 \mathrm{Mbps}$.

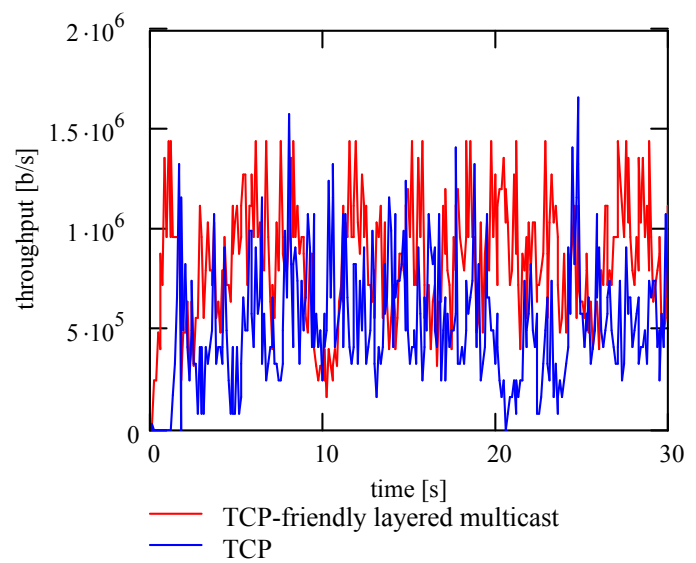

Figure 4. TCP-friendly layered multicast (6 layers) vs. FTP over TCP traffic (one out of 10 streams).

In the paper [6] is reported a similar experiment, carried out for video over TFMCC (multicast version of the TFRC) transmission. In this experiment, video over TFMCC achieves throughput of $0.846 \mathrm{Mbps}$ and FTP over TCP achieves mean throughput of 0.506 Mbps. As we can see, our solution is comparable with the TFMCC, and (in the contrast to TFMCC) is able to flexibly adapt to heterogeneous environment.

\section{Conclusions}

In the paper, ECN-capable TCP-friendly layered multicast for multimedia delivery was proposed. The proposed solution combines layered architecture with ECN-capable RTP protocol and TCP-friendly congestion control. In contrast to typical TCP-friendly solutions, which emulate TCP congestion control using TCP-throughput equation or leaky bucket algorithm, our solution directly implements an ECN-capable TCP congestion control algorithm. However, instead of RTT (impossible to estimate in heterogeneous multicast transmission), we use a modification of MRTT (multicast round trip time, applied in the WEBRC protocol).

Simulation results show that the proposed solution gives the same or better TCP-friendliness as TFRC and TFMCC protocols. Moreover, it is much better at preserving the real-time character of transmitted data. Due to its receiver-driven layered multicast architecture, it is also better at adapting to a heterogeneous environment than the TFRC and the TFMCC.

\section{Acknowledgements}

This work is partially supported by Polish Government under Grant No. N517 012 32/2108 (years 20072009).

\section{References}

[1] Chodorek, A.: "TCP and TCP-friendly protocols". Chapter of the book: Freire, M., Pereira, M. (eds.), Encyclopedia of Internet Technologies and Applications, Information Science Reference, 2008.

[2] S. Floyd, M. Handley, J. Padhye, J. Widmer, "TCP Friendly Rate Control (TFRC): Protocol Specification", $R F C$ 5348, September 2008.

[3] J. Widmer, M. Handley, "TCP-Friendly Multicast Congestion Control (TFMCC): Protocol Specification", RFC 4654, August 2006.

[4] M. Luby, V. Goyal, "Wave and Equation Based Rate Control (WEBRC) Building Block", RFC 3738, April 2004.

[5] C. Bouras, A. Gkamas, G. Kioumourtzis, "Adaptive Smooth Multicast Protocol for Multimedia Data Transmission”, SPECTS 2008, Edinburg, UK, June 2008.

[6] C. Bouras, A. Gkamas, G. Kioumourtzis, "Comparison of Single-Rate Multicast Congestion Control Protocols vs. ASMP", 16th Annual Meeting of the IEEE International Symposium on Modeling, Analysis, and Simulation of Computer and Telecommunication Systems (MASCOTS) - MASCOTS 2008, Baltimore, MD, USA.

[7] M. Zink, J. Schmitt, and R. Steinmetz, "Layer Encoded Video in Scalable Adaptive Streaming", In IEEE Transactions on Multimedia, Vol. 7, No.1, February 2005.

[8] K.Nahm; C.-C. J.Kuo, "Design and performance evaluation of tcp-friendly thin-layered video multicast scheme", Proc. of IEEE International Conference on multimedia and expo (ICME 2004), Taipei, Taiwan, June 27-30, 2004.

[9] A. Popescu, D. Constantinescu, D. Erman, D. Ilie, "A Survey of Reliable Multicast Communication", Proc. of 3rd EURO-NGI Conference on Next Generation Internet Networks, Trondheim, Norway, 21-23 May 2007.

[10] R.R. Chodorek, "A simulation of ECN-capable multicast multimedia delivery in ns-2 environment". Proc. of. 14th European Simulation, ESS'2002, October 23-26, 2002, Dresden, Germany, pp. 233-237.

[11] http://nsnam.isi.edu/nsnam/index.php/Main_Page 